\title{
HCC develops even in the early stage of chronic liver disease in elderly patients with HCV infection
}

\author{
AKIO TAKATA, RYOKO KUROMATSU, EIJI ANDO, HIDEKI IWAMOTO, NOBUYOSHI FUKUSHIMA, \\ SHUUJI SUMIE, TAKUJI TORIMURA and MICHIO SATA
}

Division of Gastroenterology, Department of Medicine, Kurume University School of Medicine, Fukuoka 830-0011, Japan

Received February 11, 2010; Accepted April 9, 2010

DOI: 10.3892/ijmm_00000459

\begin{abstract}
In recent years, the number of elderly patients with hepatocellular carcinoma (HCC) has been increasing. The aim of this study was to compare the liver function and the background factors of HCC patients with hepatitis $\mathrm{C}$ virus (HCV) infection by generation and to examine the characteristics of this disease in the elderly. A total of 1096 patients (776 men and 320 women) diagnosed with HCVrelated HCC at our institution from 1995 to 2006 were divided into 4 groups as follows: D group, 75 years of age or older; C group, 65-74 years of age; B group, 55-64 years of age; A group, 54 years of age or younger, and the liver function and other clinical characteristics were compared among these 4 groups. The average age at initial diagnosis of HCVrelated $\mathrm{HCC}$ was 66.9 years of age. The A, B, C and D groups were comprised of $87,363,514$ and 132 patients, respectively. The rate of Child-Pugh class A patients in the D group was significantly higher than that of the other groups $(\mathrm{P}<0.05)$. The average levels of ALT, TB and PT-INR in the D group were significantly lower than the levels in the other groups $(\mathrm{P}<0.05)$. The average Alb level in the $\mathrm{D}$ group was significantly higher than that in the other groups $(\mathrm{P}<0.05)$. In conclusion, we found that $\mathrm{HCV}$-related $\mathrm{HCC}$ in the elderly occurred against a background of chronic liver disease with mild inflammation and fibrosis.
\end{abstract}

\section{Introduction}

In recent years, the average age of the population of Japan has been increasing annually, and in 2006, elderly individuals over 65 years of age accounted for more than $20.8 \%$ of the entire population. The number of elderly, 65 years of age or older, who are afflicted with hepatocellular carcinoma (HCC) has also been increasing rapidly since $1990(1,2)$. The average age at initial diagnosis of $\mathrm{HCC}$ at our institution from 1986 to

Correspondence to: Dr Akio Takata, Division of Gastroenterology, Department of Medicine, Kurume University School of Medicine. 67 Asahi-machi, Kurume, Fukuoka 830-0011, Japan E-mail: oika@med.kurume-u.ac.jp

Key words: elderly patients, HCV-related HCC, mild inflammation and fibrosis, age
1993 was 60.9 years and 68.4 years in the period 2000 to 2006 and has been increasing annually (Fig. 1).

In Japan, more than $80 \%$ of HCC patients have hepatitis C virus (HCV) infection, and there are approximately 2 million patients with HCV-related chronic liver disease. It is estimated that there are 700,000 patients with undiagnosed HCV infection (3-5). The contributing factors include the spread of stimulant drugs, vaccinations and medical counter measures (injections, surgeries, blood transfusion) during the period after World War II when preventative measures against infection were inadequate. As a result, the incidence of HCC in patients with $\mathrm{HCV}$ who were infected during this period has been increasing in recent years $(1,2,4,5)$.

Generally, $60-80 \%$ of patients develop chronic hepatitis after HCV infection, while many patients present with HCC approximately 30 years after HCV infection (6-9). On the other hand, it has been reported that the majority of patients with posttransfusion chronic $\mathrm{HCV}$ infection develop HCC after the age of 60 years regardless of when they acquired the $\mathrm{HCV}$ infection (10). Moreover, with the increase in fibrosis of transplanted livers in $\mathrm{HCV}$-infected patients, it has been reported that the age of the donor is closely correlated with the rate of progression of fibrosis of the transplanted liver rather than the age of the recipient (11). Namely, the age of the patients who were infected with $\mathrm{HCV}$ is a more significant factor than the duration of the HCV infection in regards to the progression of $\mathrm{HCV}$-persistent infection and fibrosis or carcinogenesis (10). Furthermore, in recent years the number of patients in which HCC does not occur before 60 years of age but thereafter develops in patients in their 70 s or 80 s has been increasing (12-16).

Therefore, it is assumed that age is the important factor contributing to hepatocarcinogenesis of HCV-related HCC. Therefore, we divided HCV-related HCC patients into 4 groups according to age. The aim of this study was to compare the liver function and the background features of the HCVrelated HCC patients by generation, particularly focusing on the elderly group and examining the characteristics of this disease in the elderly.

\section{Materials and methods}

Patient population and experimental design. Among 1,404 patients with primary HCC consecutively diagnosed at our institution from January 1995 to December 2006, 1096 patients (776 men and 320 women) who were both HCV antibody- 


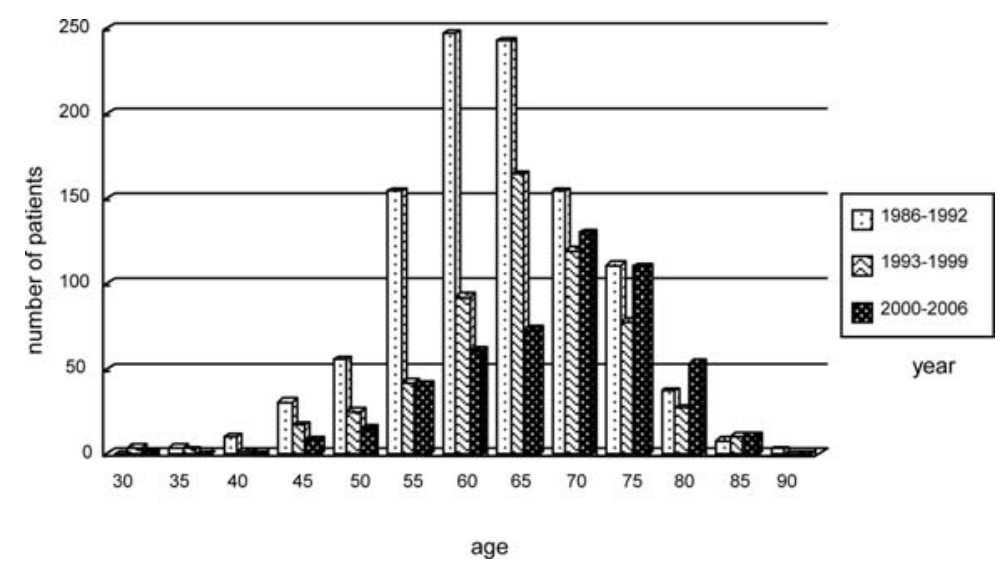

Figure 1. Changes in the average age at intitial diagnosis of HCC. The average age of onset was 60.9 years in 1986-1992, 64.9 years in 1993-1999, and 68.4 years in 2000-2006, with an annual increase in the average age.

positive and hepatitis B surface (HBs) antigen-negative were enrolled. The average age at inital diagnosis of HCC was $66 \pm 9$ years (range, 42-87). The clinical characteristics of the patients are documented in Table I. These patients were divided into 4 groups as follows: $\mathrm{D}$ group, patients $\geq 75$ years of age; C group, patients $\geq 65$ and $<75$ years of age; B group, patients $\geq 55$ and $<65$ years of age, and A group, patients $\leq 54$ years of age. Liver function and background factors were compared among the 4 groups. A liver biopsy was also performed in noncancerous areas in 18 patients in the $\mathrm{D}$ group targeted consecutively from 2005 to 2006.

HCC diagnosis. The diagnosis of HCC was based on hypervascularity, confirmed by dynamic computed tomography (CT), magnetic resonance imaging (MRI), angiography or CT angiography, when the serum levels of HCC-related tumor markers, such as $\alpha$-fetoprotein (AFP) or des- $\gamma$ carboxy prothrombin (DCP), were increased or a mass lesion was observed by ultrasonography. When a nodule was not proven to be hypervascular, percutaneous biopsy under ultrasonography was performed for confirmation of the diagnosis of HCC, and thereafter a pathological study using hematoxylin and eosin staining was carried out. The fibrosis staging scores and activity grades were assigned according to the criteria of Desmet and colleagues (17) and the French METAVIR Cooperative Study (18-20). Staging was defined as F0 (no fibrosis), F1 (mild fibrosis), F2 (moderate fibrosis), F3 (severe fibrosis), or F4 (cirrhosis), and grading was defined as A0 (no activity), A1 (mild activity), A2 (moderate activity), or A3 (severe activity). Three pathologists independently evaluated the disease stage and grade. Portal vein tumor thrombosis (PVTT) was defined as a protrusion of the tumor into the first and/or second branch, or into the main trunk of the portal vein. Tumor stage was classified according to the International Union Against Cancer (UICC) TNM classification.

Factors evaluated in the analysis. The following 19 factors were compared: gender, a history of blood transfusion, a history of habitual drinking, underlying diseases except for liver diseases, gastro-esophageal varices, Child-Pugh classification, serum albumin (Alb), serum total bilirubin (TB), prothrombin time-international normalized ratio (PT-INR),
Table I. Profile of the 1096 patients at initial diagnosis of HCV-related HCC.

\begin{tabular}{lc}
\hline Age (range) & $66.9 \pm 7.6(42-87)$ \\
Gender (male/female) & $776 / 320$ \\
Alcohol consumption (none/light/heavy) & $392 / 371 / 333$ \\
Underlying disease (none/single/multiple) $^{\text {a }}$ & $471 / 436 / 189$ \\
Gastro-esophageal varices (none/small/large) $^{\mathrm{a}}$ & $436 / 393 / 240$ \\
Blood transfusion (yes/no) $^{\text {Child-Pugh classification (A/B/C) }}$ & $377 / 719$ \\
Alb (g/dl) & $751 / 290 / 55$ \\
PT-INR & $3.48 \pm 0.47$ \\
TB (mg/dl) & $1.17 \pm 0.15$ \\
ALT (U/l) & $1.17 \pm 0.66$ \\
PLT (x104/ 1$)$ & $64.4 \pm 39.0$ \\
HA (ng/ml) & $10.8 \pm 5.54$ \\
ICG R15 $(\%)$ & $363 \pm 385$ \\
\hline
\end{tabular}

Date are expressed as the median range \pm standard deviation (SD). ${ }^{a}$ Twenty seven patients did not undergo endoscopic examination. Alb, serum albumin; PT-INR, prothrombin time-international normalized ratio; TB, serum total bilirubin; ALT, serum alanine aminotransferase; PLT, platelet count; HA, hyaluronic acid; ICG R15, indocyanine green retention rate at $15 \mathrm{~min}$.

serum alanine aminotransferase (ALT), platelet count (PLT), indocyanine green retention rate at $15 \mathrm{~min}$ (ICG R15), serum hyaluronic acid (HA), number of tumors, tumor size, portal vein tumor thrombosis (PVTT), tumor stage, $\alpha$-fetoprotein (AFP) and extra-hepatic metastasis. The values at initial diagnosis of HCC were used for Alb, TB, PT-INR, ALT, PLT, ICG R15 and HA. Chronic hepatitis was included as ChildPugh class A. Gastro-esophageal varices were classified into three types according to the endoscopic form factor grade: none, no varix; small, form 1 or 2; large, form 3, based on the classification of the Japanese Research Society for Portal Hypertension (21). The underlying diseases included hypertension, diabetes mellitus, cerebrovascular damage, cardiac disorder, renal disorder and cancer of other organs, and these were divided into three groups: none, no underlying disease; single, underlying disease by one disorder; multiple, 


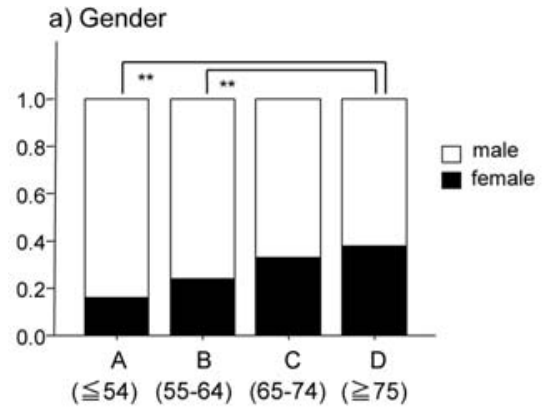

c) Underlying diseases

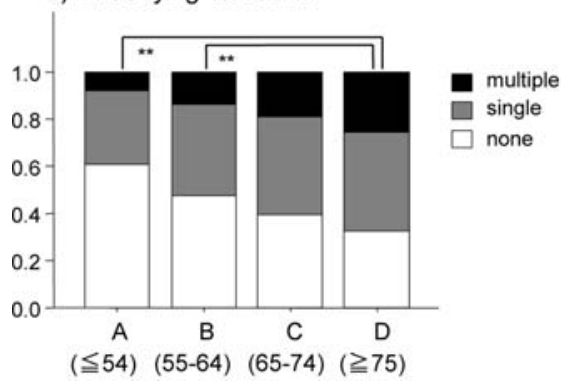

b) A history of habitual drinking

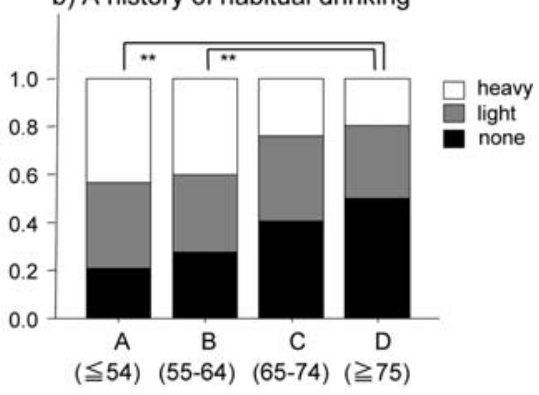

d) A history of blood transfusion

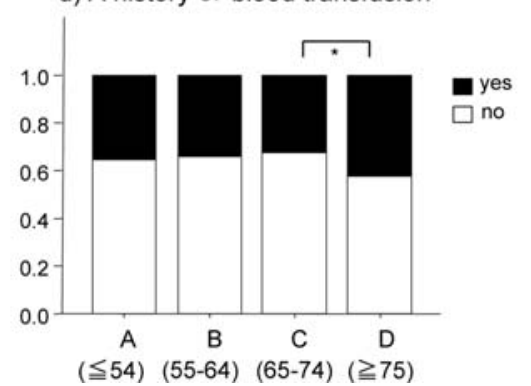

Figure 2. The age at initial diagnosis of HCC and background factors. The number of female patients, the patients without a history of habitual drinking and the patients with underlying diseases increased with age. (a) Concerning gender, the number of female patients in the D group was significantly higher than that in groups $\mathrm{A}$ and $\mathrm{B}\left({ }^{* *} \mathrm{P}<0.01\right)$. (b) The number of patients without a history of habitual drinking in the $\mathrm{D}$ group was significantly higher than that in the $\mathrm{A}$ and $\mathrm{B}$ groups $\left({ }^{* *} \mathrm{P}<0.01\right)$. (c) The number of patients with underlying diseases in the $\mathrm{D}$ group was significantly higher than that in the $\mathrm{A}$ and $\mathrm{B}$ groups $\left({ }^{* *} \mathrm{P}<0.01\right)$. (d) A history of blood transfusion was noted most frequently in the $\mathrm{D}$ group and was significantly higher than that in the $\mathrm{A}$ group $\left({ }^{*} \mathrm{P}<0.05\right)$. ${ }^{*} \mathrm{P}<0.05,{ }^{* *} \mathrm{P}<0.01$ between the indicated groups.

underlying diseases by more than two disorders. A history of habitual drinking was classified into three groups: none, nondrinker; light, $<84 \mathrm{~g} /$ day of ethanol; heavy, $>84 \mathrm{~g} /$ day of ethanol for $>5$ years. The number of tumors was divided into two groups: solitary and multiple tumors. Tumor size was divided into two groups: those measuring $\leq 3 \mathrm{~cm}$ and $>3 \mathrm{~cm}$ in size. The AFP level was divided into three categories: $\leq 20$, 21-200 and >200 ng/ml.

Statistical analysis. Data are expressed as the mean \pm standard deviation (SD). Data regarding Alb, TB, PT-INR, ALT, PLT, ICG R15 and HA levels were analyzed by oneway analysis of variance followed by the Dunnett's multiple comparison of means test. A Logistics regression analysis was used to analyze the effects of the background factors (gender, a history of blood transfusion, a history of habitual drinking, underlying diseases, degree of gastro-esophageal varices, Child-Pugh classification) and the tumor features (number of tumors, tumor size, PVTT, tumor stage, AFP, extrahepatic metastasis) on age at the initial diagnosis of HCC. The background factors and the tumor features were individually chosen as outcome variables, and the age at initial diagnosis of HCC divided into four groups were dummy coded and used as predictor variables. The statistical analysis was performed using SPSS for Windows (version 12.0). $\mathrm{P}<0.05$ was considered to be statistically significant.

\section{Results}

Comparison of background factors. Among the 1096 patients, $132(12 \%)$ were classified in the D group (patients $\geq 75$ years of age), with the oldest patient being 87 years of

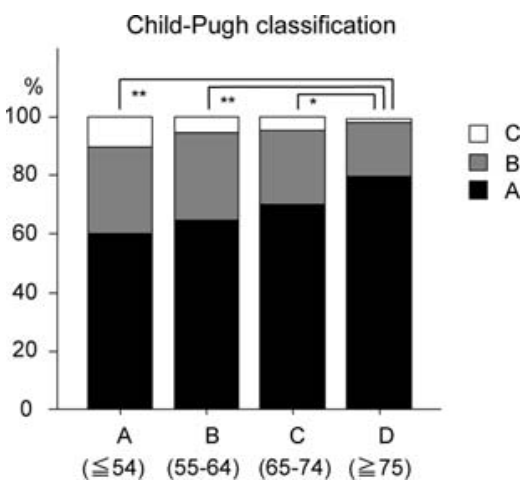

Figure 3. The age at initial diagnosis of HCC and Child-Pugh classification. The percentages of Child-Pugh class A patients were 59.8, 64.5, 70.1 and $79.5 \%$ in the A, B, C and D groups, respectively. The rate of Child-Pugh class A patients increased with age, and the rate of Child-Pugh class A patients in the $\mathrm{D}$ group was significantly higher than the rate in the other groups (D vs. A and $\mathrm{B},{ }^{* *} \mathrm{P}<0.01$; D vs. $\mathrm{C},{ }^{*} \mathrm{P}<0.05$ ). ${ }^{*} \mathrm{P}<0.05,{ }^{* *} \mathrm{P}<0.01$ between the indicated groups.

age. The number of female patients increased with age, and the male:female patient ratio in the $\mathrm{D}$ group was 82:50. As a result, the number for female patients in the D group was significantly higher than that in the A and B groups (patients $<65$ years of age) $(\mathrm{P}<0.01)$ (Fig. 2a). The number of patients without a history of habitual drinking increased with age, and that of the $\mathrm{D}$ group was significantly higher than that in the $\mathrm{A}$ and $\mathrm{B}$ groups $(\mathrm{P}<0.01)$ (Fig. 2b). The number of patients with underlying diseases increased with age, and that of the $\mathrm{D}$ group was significantly higher than that in the A and B groups $(\mathrm{P}<0.01)$ (Fig. 2c). A history of blood transfusion was 

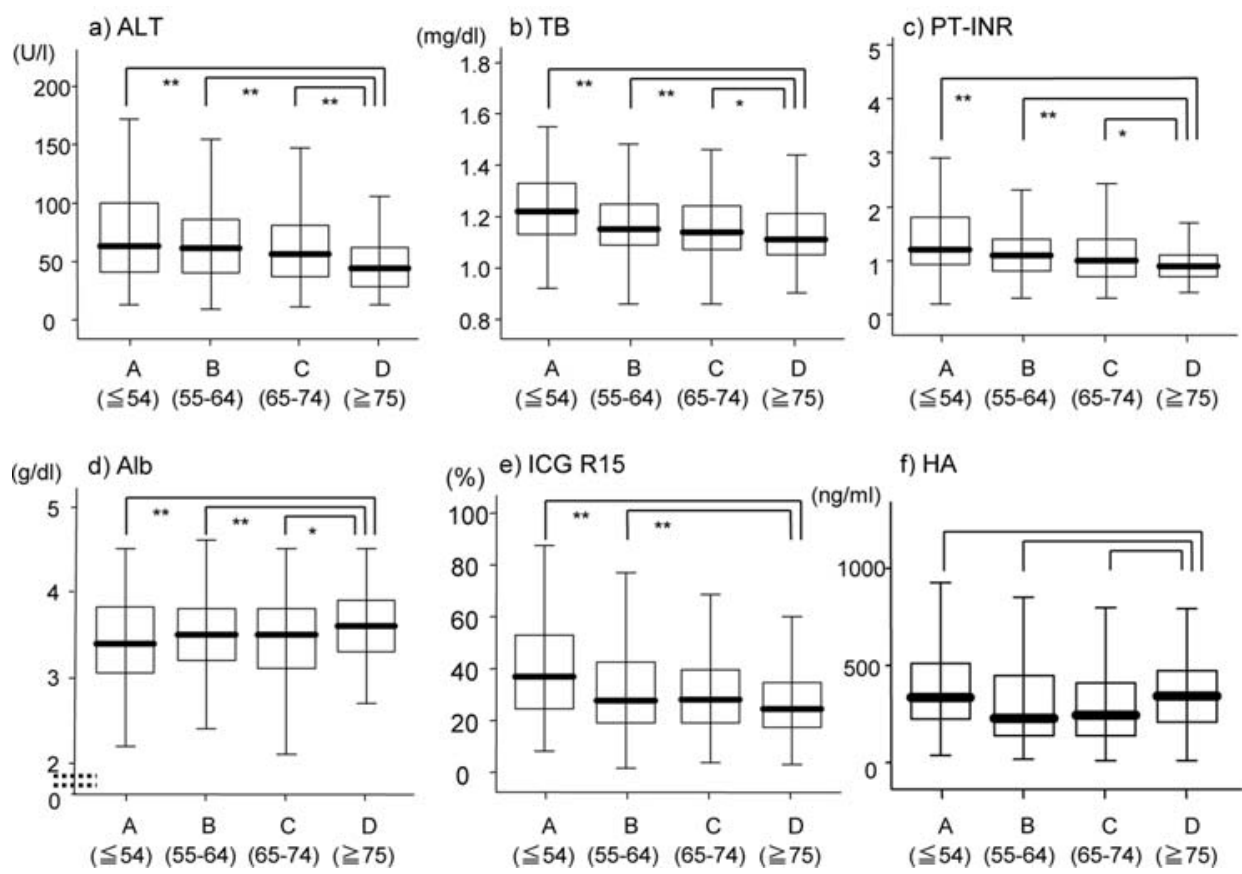

f) $H A$

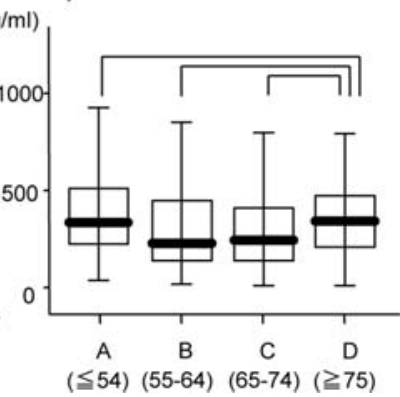

Figure 4. A comparison of age at initial diagnosis of HCC and liver functions. The average values of (a) ALT, (b) TB and (c) PT-INR in the D group were significantly lower than the values in the other groups (ALT: D vs. A, B and C, ${ }^{* *} \mathrm{P}<0.01$; TB and PT-INR: D vs. A and $\mathrm{B},{ }^{* *} \mathrm{P}<0.01, \mathrm{D}$ vs. C, ${ }^{*} \mathrm{P}<0.05$ ). The average value of (d) Alb in the D group was significantly higher than that in the other groups (D vs. A and $\mathrm{B},{ }^{* * *} \mathrm{P}<0.01 ; \mathrm{D}$ vs. $\mathrm{C},{ }^{*} \mathrm{P}<0.05$ ). The average value of (e) ICG R15 in the D group was significantly lower than the value in the A and B groups (D vs. A and B, ${ }^{* *} \mathrm{P}<0.01$ ). The average value of (f) HA did not show a significant difference. ${ }^{*} \mathrm{P}<0.05,{ }^{* *} \mathrm{P}<0.01$ between the indicated groups.
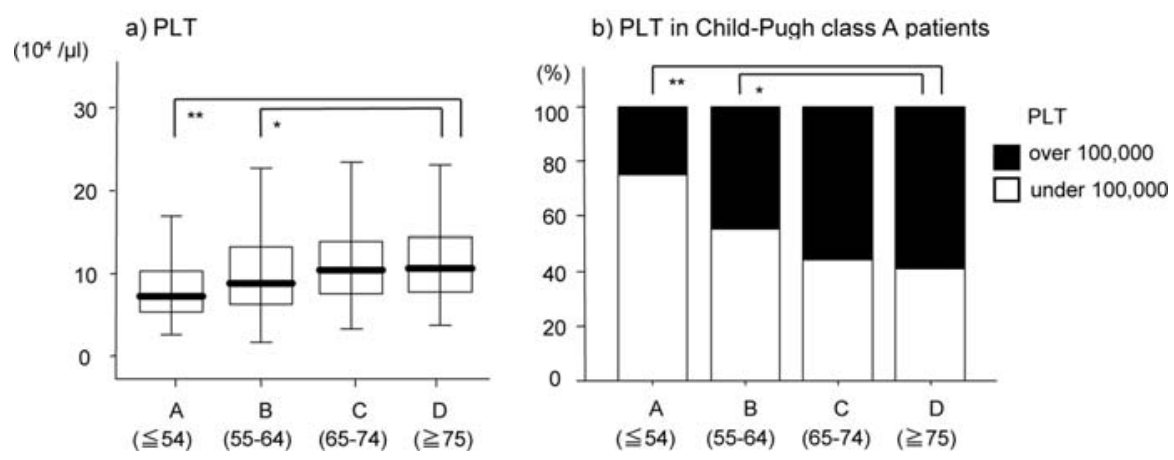

Figure 5. Comparison of age at initial diagnosis of HCC and PLT, and PLT in Child-Pugh class A patients. (a) The average value of PLT in the D group was significantly higher than the value in the $\mathrm{A}\left({ }^{* *} \mathrm{P}<0.01\right)$ and $\mathrm{B}\left({ }^{*} \mathrm{P}<0.05\right)$ groups. (b) The rate of 100,000 or more of PLT in Child-Pugh class A patients of the $\mathrm{D}$ group was significantly higher than that in the $\mathrm{A}\left({ }^{* *} \mathrm{P}<0.01\right)$ and $\mathrm{B}\left({ }^{*} \mathrm{P}<0.05\right)$ groups. ${ }^{*} \mathrm{P}<0.05,{ }^{* *} \mathrm{P}<0.01$ between the indicated groups.

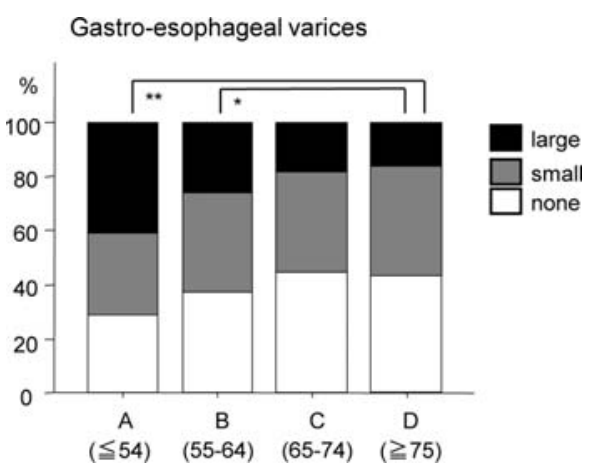

Figure 6. The age at initial diagnosis of $\mathrm{HCC}$ and the endoscopic form factor grade of varices. The number of patients with large varices was the lowest in the $\mathrm{D}$ group and the highest in the A group. The number of patients with large varices in the $\mathrm{D}$ group was significantly lower than that in the $\mathrm{A}\left({ }^{* *} \mathrm{P}<0.01\right)$ and $\mathrm{B}\left({ }^{*} \mathrm{P}<0.05\right)$ groups. ${ }^{*} \mathrm{P}<0.05,{ }^{* *} \mathrm{P}<0.01$ between the indicated groups. noted most frequently in the D group, and it was significantly higher than that in the A group $(\mathrm{P}<0.05)$ (Fig. 2d).

Comparison of liver function. The percentages of Child-Pugh class A patients were $59.8,64.5,70.1$ and $79.5 \%$, in the A, B, $\mathrm{C}$ and $\mathrm{D}$ groups, respectively. Therefore, the percentage of Child-Pugh class A patients increased with age, and the percentage of Child-Pugh class A patients in the D group was higher than the other groups (D vs. A, B, P<0.01; D vs. C, $\mathrm{P}<0.05$ ) (Fig. 3). The average values of ALT, TB and PTINR were lower with age, and these average values in the $\mathrm{D}$ group were significantly lower than those in the other groups (ALT: D vs. A, B and C, P<0.01; TB, PT-INR: D vs. A and $\mathrm{B}, \mathrm{P}<0.01$; D vs. $\mathrm{C}, \mathrm{P}<0.05$ ) (Fig. $4 \mathrm{a}-\mathrm{c})$. The average value of Alb in the $\mathrm{D}$ group was significantly higher than that in the other groups ( $\mathrm{D}$ vs. A and $\mathrm{B}, \mathrm{P}<0.01$; D vs. $\mathrm{C}, \mathrm{P}<0.05$ ) 
Table II. Characteristics of the HCC tumors of the 1096 patients at initial diagnosis of HCV-related HCC.

\begin{tabular}{|c|c|c|c|c|c|}
\hline & $\begin{array}{c}\text { A group (87) } \\
42-54 \text { years of age }\end{array}$ & $\begin{array}{l}\text { B group }(363) \\
55-64 \text { years }\end{array}$ & $\begin{array}{l}\text { C group }(514) \\
65-74 \text { years }\end{array}$ & $\begin{array}{l}\text { D group (132) } \\
75-87 \text { years }\end{array}$ & P-value \\
\hline Number of tumors & & & & & $<0.05$, D vs. C \\
\hline Solitary & 37 & 156 & 217 & 59 & \\
\hline Multiple & 50 & 207 & 297 & 73 & \\
\hline Tumor size & & & & & $<0.05, \mathrm{D}$ vs. $\mathrm{A}$ and $\mathrm{B}$ \\
\hline$\leq 3 \mathrm{~cm}$ & 61 & 238 & 312 & 73 & \\
\hline$>3 \mathrm{~cm}$ & 26 & 125 & 202 & 59 & \\
\hline PVTT & & & & & NS \\
\hline Present & 7 & 45 & 66 & 11 & \\
\hline Absent & 80 & 318 & 448 & 121 & \\
\hline TNM staging & & & & & NS \\
\hline $\mathrm{I}$ & 14 & 88 & 106 & 20 & \\
\hline II & 37 & 120 & 171 & 51 & \\
\hline III & 30 & 107 & 174 & 49 & \\
\hline IV & 6 & 48 & 63 & 12 & \\
\hline AFP (ng/ml) & & & & & NS \\
\hline$\leq 20$ & 28 & 120 & 185 & 53 & \\
\hline $21-200$ & 41 & 158 & 183 & 42 & \\
\hline$>200$ & 18 & 85 & 146 & 37 & \\
\hline Extrahepatic metastasis & & & & & NS \\
\hline Yes & 2 & 20 & 18 & 3 & \\
\hline No & 85 & 343 & 496 & 129 & \\
\hline
\end{tabular}

PVTT, portal vein tumor thrombosis; AFP, $\alpha$-fetoprotein.

(Fig. 4d). The average value of ICG R15 was the lowest in the D group, and the average value of ICG R15 in the D group was significantly lower than that in the $\mathrm{A}$ and $\mathrm{B}$ groups (D vs. $\mathrm{A}$ and $\mathrm{B}, \mathrm{P}<0.01$ ) (Fig. 4e). The average value of HA was the lowest in the $\mathrm{C}$ group, thus indicating a strong variation and no significant difference (Fig. 4f). The average value of PLT was the highest in the D group, and the average value of PLT in the $\mathrm{D}$ group was significantly higher than that in the $\mathrm{A}$ and $\mathrm{B}$ groups (D vs. A, $\mathrm{P}<0.01$; D vs. B, $\mathrm{P}<0.05$ ) (Fig. 5a). When the Child-Pugh class A patients were divided per $100,000 / \mu 1$ of PLT and compared, the rate of 100,000 or more in the $\mathrm{D}$ group increased with age, and it was higher than the $\mathrm{A}$ and $\mathrm{B}$ groups (D vs. A, $\mathrm{P}<0.01$; D vs. $\mathrm{B}, \mathrm{P}<0.05$ ) (Fig. 5b). The number of patients with large varices decreased with age, and the number of patients with large varices in the $\mathrm{D}$ group was lower than that in the $\mathrm{A}$ and $\mathrm{B}$ groups (D vs. A, $\mathrm{P}<0.01$; D vs. B, $\mathrm{P}<0.05)$ (Fig. 6).

Comparison of characteristics of the HCC tumors. The number of patients with small HCCs $\leq 3 \mathrm{~cm}$ in the D group was lower than that in the A and B groups (D vs. A and B, $\mathrm{P}<0.05)$, and the number of patients with solitary HCC tumor in the $\mathrm{D}$ group was higher than that in the $\mathrm{C}$ group (D vs. C, $\mathrm{P}<0.05$ ) (Table II). There were no statistical differences in the prevalence of PVTT, tumor stage, AFP or extrahepatic metastasis.
Pathological findings of liver biopsies from noncancerous areas. The pathological findings of patients for whom liver biopsies from noncancerous areas were performed are documented in Table III. Four of the 18 patients showed liver cirrhosis with activity $\geq 2$ and fibrosis 4 in the Desmet classification. Fourteen of the 18 patients showed chronic hepatitis that was milder than activity 2 and fibrosis 3 in the Desmet classification.

\section{Discussion}

An elder is generally defined as anyone 65 years of age or older, but this definition is not standardized worldwide. In Japan, the average age at initial diagnosis of HCC is 65 years or older $(13,14,22)$. Therefore, it is not appropriate to draw the dividing line at 65 years of age in order to define the characteristics of HCC that presents at old age (23). On the other hand, it has been reported that HCV-related HCC which develops in individuals in their 40s exhibits different characteristics from HCV-related HCC which develops in individuals 50 years of age or older, since HCC in individuals in their $40 \mathrm{~s}$ is independently related to heavy drinking and the presence of $\mathrm{HBV}$ coinfection (24). It is therefore not appropriate that younger patients in their 40s and older patients who are 65 years of age or older are treated as a similar group, even when HCC patients are divided by a line drawn at 70 years 
Table III. Pathological findings of liver biopsies from non-cancerous areas and the liver function of 18 patients with HCC 75 years of age or older.

\begin{tabular}{rcccrcrrrrr}
\hline No. & Age & Gender & $\begin{array}{c}\text { Histology } \\
\text { Desmet classification }\end{array}$ & $\begin{array}{c}\text { PLT } \\
\left(\mathrm{x} 10^{4} / \mu \mathrm{l}\right)\end{array}$ & $\begin{array}{r}\text { ALT } \\
(\mathrm{U} / \mathrm{l})\end{array}$ & $\begin{array}{c}\text { TB } \\
(\mathrm{mg} / \mathrm{dl})\end{array}$ & PT-INR & $\begin{array}{c}\text { Alb } \\
(\mathrm{g} / \mathrm{dl})\end{array}$ & $\begin{array}{r}\text { ICG R15 } \\
(\%)\end{array}$ & $\begin{array}{c}\text { HA } \\
(\mathrm{ng} / \mathrm{ml})\end{array}$ \\
\hline 1 & 78 & $\mathrm{~F}$ & A1F1 & 9.4 & 87 & 1.10 & 1.09 & 3.6 & 30.6 & 287 \\
2 & 80 & $\mathrm{M}$ & A2F4 & 7.9 & 79 & 0.70 & 1.03 & 3.3 & 14.3 & 237 \\
3 & 82 & $\mathrm{~F}$ & A2F2 & 37.5 & 37 & 0.50 & 0.97 & 4.1 & 9.7 & 123 \\
4 & 85 & $\mathrm{~F}$ & A2F2 & 8.3 & 28 & 0.50 & 1.06 & 4.1 & 16.8 & 470 \\
5 & 75 & $\mathrm{M}$ & A2F2 & 13.8 & 44 & 0.90 & 1.03 & 3.5 & 28.9 & 265 \\
6 & 77 & $\mathrm{M}$ & A2F2 & 17.5 & 64 & 1.30 & 1.13 & 4.0 & 30.3 & 225 \\
7 & 75 & $\mathrm{M}$ & A2F1 & 12.5 & 22 & 0.90 & 0.97 & 4.1 & 14.2 & 62 \\
8 & 80 & $\mathrm{~F}$ & A2F2 & 15.3 & 54 & 1.00 & 1.12 & 3.4 & 45.7 & 1340 \\
9 & 76 & $\mathrm{M}$ & A2F4 & 8.3 & 62 & 0.80 & 1.18 & 3.5 & 24.5 & 557 \\
10 & 87 & $\mathrm{M}$ & A2F2 & 8.1 & 43 & 0.90 & 1.05 & 3.8 & 22.1 & 503 \\
11 & 78 & $\mathrm{M}$ & A1F1 & 16.3 & 33 & 0.95 & 1.05 & 4.5 & 16.8 & 126 \\
12 & 76 & $\mathrm{M}$ & A2F4 & 13.9 & 77 & 1.20 & 1.16 & 3.8 & 16.2 & 405 \\
13 & 75 & $\mathrm{M}$ & A3F4 & 8.9 & 33 & 1.40 & 1.16 & 3.7 & 48.2 & 619 \\
14 & 76 & $\mathrm{~F}$ & A1F2 & 8.0 & 52 & 0.90 & 1.08 & 3.7 & 18.0 & 260 \\
15 & 82 & $\mathrm{~F}$ & A2F3 & 11.0 & 37 & 2.20 & 1.20 & 3.1 & 23.8 & 1360 \\
16 & 76 & $\mathrm{~F}$ & A2F2 & 11.9 & 45 & 0.70 & 1.02 & 4.2 & 9.6 & 353 \\
17 & 76 & $\mathrm{M}$ & A1F2 & 13.3 & 40 & 0.40 & 1.04 & 3.4 & 25.9 & 732 \\
18 & 77 & $\mathrm{M}$ & A2F3 & 10.1 & 54 & 1.02 & 1.01 & 4.1 & 45.2 & 163 \\
Average & 78.3 & & & 12.9 & 49.5 & 0.97 & 1.08 & 3.77 & 24.5 & 449 \\
\hline
\end{tabular}

PLT, platelet count; ALT, serum alanine aminotransferase; TB, serum total bilirubin; PT-INR, prothrombin time-international normalized ratio; Alb, serum albumin; ICG R15, indocyanine green retention rate at $15 \mathrm{~min}$; HA, serum hyaluronic acid.

of age as reported in previous studies $(14,15,25,26)$. We therefore divided the elderly into the $\mathrm{C}$ group $(>65$ years of age and $<75$ years) and the $\mathrm{D}$ group ( $\geq 75$ years of age), and the nonelderly into the A group ( $<54$ years of age) and the B group ( $>55$ and $<65$ years of age) to create 4 groups. The clinical characteristics of HCC in each group were thus examined in further detail by comparing each group, while particularly focusing on the $\mathrm{D}$ group.

Consequently, the most significant characteristic in patients with HCV-related HCC in the D group was that hepatic reserve was maintained and HCC occurred against a background of liver disorder with mild inflammation and fibrosis. In our study, levels of ALT and TB, which are well-known markers of inflammatory necrosis in the liver, were significantly lower in the D group than in all of the other groups. Notably, some studies found that alcohol promotes the progression of background chronic liver disease and consequently enhances carcinogenesis of the liver or that alcohol directly promotes carcinogenesis of the liver (27-29). In our study, the A group of younger HCC patients included many heavy drinkers of alcohol with severe inflammation in addition to high values of ALT and TB, as was previously found by Shimauchi et al (24). This finding corroborates reports that $\mathrm{HCC}$ frequently occurs in association with severe inflammation (30). Conversely, it was assumed to be one of the main reason that the $\mathrm{D}$ group had many patients with low values of ALT and TB, since the D group included many patients who did not habitually consume alcohol.
It has been reported that in chronic liver disease, changes in the platelet count correlate with the degree of fibrosis in liver histology (31-33). As a result, the platelet count is regarded as a marker for fibrosis. In our study, the platelet count in the $\mathrm{D}$ group was significantly higher than that in the $\mathrm{A}$ and $\mathrm{B}$ groups (younger than 65 years of age), and the D group in particular showed the highest platelet count of 100,000 or more in the Child-Pugh class A patients. The study of 18 patients in the $\mathrm{D}$ group was assessed by means of a liver biopsy, and the background liver showed chronic hepatitis of less than F3 except for 4 cases of F4, thus indicating that many patients with mild fibrosis had not advanced to liver cirrhosis.

Gastro-esophageal varices are the most common clinical manifestation of portal hypertension in patients with liver cirrhosis. Nakayama et al (34) reported that as the endoscopic form factor grade of varix increased, the incidence of the occurrence of HCC also increased, and in particular, a form 3 (large size) factor of varices was an independent predictor for HCC. In our study, many of the patients in the D group either did not have varices or only had small varices, and also the number of patients with large varices in the D group was significantly smaller than the non-elderly groups (younger than 65 years of age). Therefore, it was assumed that many of the patients in the $\mathrm{D}$ group had not advanced to liver cirrhosis, and many cases of HCC had thus occurred against a background of chronic liver disease that was not conducive to carcinogenesis. 
Alb, PT-INR and ICG R15 values are well-known markers of hepatic reserve in the liver. In our study, the average values of Alb, PT-INR and ICG R15 in the D group were higher than in all of the other groups, showing the highest rate in the Child-Pugh class A patients. Generally, it has been reported that a decreased number of hepatocytes and decreased liver weight due to aging generally results in a decreased regeneration capacity of hepatocytes and a decreased hepatic blood flow (35-38). In addition, it has been suggested that the elderly often have a latent nutrient disturbance or metabolic anomalies, thus causing a declining trend in Alb and ICG R15 $(39,40)$. However, in our study, many cases of HCC in the D group were present along with low values of ALT and TB, unlike the non-elderly group. Therefore, it appears that the progression of chronic liver disease was not promoted, and the hepatic reserve was consequently well-maintained in the elderly HCC group, despite the normal decline in the physiological liver function with age. As noted above, many cases of HCC in the elderly occur against a background of chronic liver disease with mild inflammation and fibrosis, and it is assumed that there are some characteristic factors which contribute to hepatocarcinogenesis in the elderly.

In recent years, age has become a point of focus in the progression of $\mathrm{HCV}$-persistent infection and fibrosis or carcinogenesis. Mahmood et al (41) reported that age was the most important factor contributing to a high value of reactive oxygen metabolites in the blood associated with HCV-related chronic liver disease. Moreover, the involvement of oxidative stress has been previously reported in aging itself, which is believed to cause a decrease in various organ functions and immunity (42). The elderly are susceptible to oxidative stress due to a decrease in the SOD value related to the antioxidation mechanism and the decrease in NK cell activity $(42,43)$. Therefore, hepatocarcinogenesis in the elderly may occur in association with only mild inflammation or fibrosis.

In addition, an increase in the rate of women is cited as one of the characteristics of HCC of the elderly. It has been reported that sex hormones such as estrogen and immune response play an important role in hepatocarcinogenesis $(44,45)$. Since the levels of sex hormones decrease in ageing women, the risk of the incidence of HCC in elderly females increases.

In conclusion, we found that $\mathrm{HCV}$-related $\mathrm{HCC}$ in the elderly occurred against a background of chronic liver disease with mild inflammation and fibrosis.

\section{References}

1. Tanaka H, Imai Y, Hiramatsu N, et al: Declining incidence of hepatocellular carcinoma in Osaka, Japan, from 1990 to 2003. Ann Intern Med 148: 820-826, 2008.

2. Taura N, Hamasaki K, Nakao K, et al: Aging of patients with hepatitis C virus-associated hepatocellular carcinoma: Longterm trends in Japan. Oncol Rep 16: 837-843, 2006.

3. Yano $M$, Yatsuhashi $H$, Inoue $O$, Inokuchi $K$ and Koga $M$ : Epidemiology and long term prognosis of hepatitis $\mathrm{C}$ virus infection in Japan. Gut 34: S13-S16, 1993.

4. Moriya T, Koyama T, Tanaka J, Mishiro S and Yoshizawa H: Epidemiology of hepatitis C virus in Japan. Intervirology 42: $153-158,1999$

5. Tsukuma H, Tanaka H, Ajiki W and Oshima A: Liver cancer and its prevention. Asian Pac J Cancer Prev 6: 244-250, 2005.

6. Gerlach JT, Diepolder HM, Zachoval R, et al: Acute hepatitis C: high rate of both spontaneous and treatment-induced viral clearance. Gastroenterology 125: 80-88, 2003.
7. Ikeda K, Saitoh S, Koida I, et al: A multivariate analysis of risk factors for hepatocellular carcinogenesis: a prospective observation of 795 patients with viral and alcoholic cirrhosis. Hepatology 18: 47-53, 1993.

8. Kiyosawa K, Umemura T, Ichijo T, et al: Hepatocellular carcinoma: recent trends in Japan. Gastroenterology 127: S17-S26, 2004.

9. Yatsuhashi $\mathrm{H}$ and Yano M: Natural history of chronic hepatitis C. J Gastroenterol Hepatol 15: E111-E116, 2000.

10. Hamada H, Yatsuhashi H, Yano K, et al: Impact of aging on the development of hepatocellular carcinoma in patients with posttransfusion chronic hepatitis C. Cancer 95: 331-339, 2002.

11. Wali M, Harrison RF, Gow PJ and Mutimer D: Advancing donor liver age and rapid fibrosis progression following transplantation for hepatitis C. Gut 51: 248-252, 2002.

12. Dohmen K, Shirahama M, Shigematsu H, Irie K and Ishibashi H: Optimal treatment strategy for elderly patients with hepatocellular carcinoma. J Gastroenterol Hepatol 19: 859-865, 2004.

13. Ohishi W, Kitamoto M, Aikata H, et al: Impact of aging on the development of hepatocellular carcinoma in patients with hepatitis C virus infection in Japan. Scand J Gastroenterol 38: 894-900, 2003

14. Teratani T, Ishikawa T, Shiratori Y, et al: Hepatocellular carcinoma in elderly patients: beneficial therapeutic efficacy using percutaneous ethanol injection therapy. Cancer 95: 816-823, 2002.

15. Ferrero A, Vigano L, Polastri R, et al: Hepatectomy as treatment of choice for hepatocellular carcinoma in elderly cirrhotic patients. World J Surg 29: 1101-1105, 2005.

16. Tsukioka G, Kakizaki S, Sohara N, et al: Hepatocellular carcinoma in extremely elderly patients: an analysis of clinical characteristics, prognosis and patient survival. World J Gastroenterol 12: 48-53, 2006.

17. Desmet VJ, Gerber M, Hoofnagle JH, Manns M and Scheuer PJ: Classification of chronic hepatitis: diagnosis, grading and staging. Hepatology 19: 1513-1520, 1994.

18. Poynard T, Bedossa P and Opolon P: Natural history of liver fibrosis progression in patients with chronic hepatitis $\mathrm{C}$. The OBSVIRC, METAVIR, CLINIVIR, and DOSVIRC groups. Lancet 349: 825-832, 1997.

19. Bedossa P and Poynard T: An algorithm for the grading of activity in chronic hepatitis C. The METAVIR Cooperative Study Group. Hepatology 24: 289-293, 1996.

20. The French METAVIR Cooperative Study Group: Intraobserver and interobserver variations in liver biopsy interpretation in patients with chronic hepatitis C. Hepatology 20: 15-20, 1994.

21 . The general rules for recording endoscopic findings on esophageal varices. Jpn J Surg 10: 84-87, 1980.

22. Dohmen K, Shigematsu H, Irie $\mathrm{K}$ and Ishibashi $\mathrm{H}$ : Trends in clinical characteristics, treatment and prognosis of hepatocellular carcinoma. Hepatogastroenterology 50: 1872-1877, 2003.

23. Zhou L, Rui JA, Wang SB, et al: Clinicopathological features, post-surgical survival and prognostic indicators of elderly patients with hepatocellular carcinoma. Eur J Surg Oncol 32: 767-772, 2006

24. Shimauchi Y, Tanaka M, Koga K, et al: Clinical characteristics of patients in their 40s with HCV antibody-positive hepatocellular carcinoma. Alcohol Clin Exp Res 24: 64S-67S, 2000

25. Nomura F, Ohnishi K, Honda M, Satomura Y, Nakai T and Okuda K: Clinical features of hepatocellular carcinoma in the elderly: a study of 91 patients older than 70 years. Br J Cancer 70: 690-693, 1994.

26. Hanazaki K, Kajikawa S, Shimozawa N, et al: Hepatic resection for hepatocellular carcinoma in the elderly. J Am Coll Surg 192: 38-46, 2001.

27. Yamagishi Y, Horie Y, Kajihara M, et al: Hepatocellular carcinoma in heavy drinkers with negative markers for viral hepatitis. Hepatol Res 28: 177-183, 2004.

28. Sata M, Fukuizumi K, Uchimura Y, et al: Hepatitis C virus infection in patients with clinically diagnosed alcoholic liver diseases. J Viral Hepat 3: 143-148, 1996.

29. Corrao G and Arico S: Independent and combined action of hepatitis $\mathrm{C}$ virus infection and alcohol consumption on the risk of symptomatic liver cirrhosis. Hepatology 27: 914-919, 1998.

30. Tarao K, Rino Y, Ohkawa S, et al: Association between high serum alanine aminotransferase levels and more rapid development and higher rate of incidence of hepatocellular carcinoma in patients with hepatitis $\mathrm{C}$ virus-associated cirrhosis. Cancer 86: $589-595,1999$. 
31. Ono E, Shiratori Y, Okudaira T, et al: Platelet count reflects stage of chronic hepatitis C. Hepatol Res 15: 192-200, 1999.

32. Matsumura H, Moriyama M, Goto I, Tanaka N, Okubo H and Arakawa Y: Natural course of progression of liver fibrosis in Japanese patients with chronic liver disease type C - a study of 527 patients at one establishment. J Viral Hepat 7: 268-275, 2000.

33. Karasu Z, Tekin F, Ersoz G, et al: Liver fibrosis is associated with decreased peripheral platelet count in patients with chronic hepatitis B and C. Dig Dis Sci 52: 1535-1539, 2007.

34. Nakayama H, Masuda H, Miyake H, Takayama $T$ and Yokoyama E: Endoscopic prediction of hepatocellular carcinoma by evaluation of bleeding esophageal varices. Digestion 70: 233-239, 2004.

35. Okudaira M, Ikawa N, Yasuhara M, Kumagai T and Kurosu K: Liver weight of adult Japanese, especially recent weight values. Hepatol Res 18: 95-103, 2000.

36. Zoli M, Iervese T, Abbati S, Bianchi GP, Marchesini G and Pisi E: Portal blood velocity and flow in aging man. Gerontology 35: 61-65, 1989.

37. Mooney H, Roberts R, Cooksley WG, Halliday JW and Powell LW: Alterations in the liver with ageing. Clin Gastroenterol 14: 757-771, 1985.

38. Jansen PL: Liver disease in the elderly. Best Pract Res Clin Gastroenterol 16: 149-158, 2002.
39. Wynne HA, Mutch E, Williams FM, James OF, Rawlins MD and Woodhouse KW: The relation of age to the acute effects of ethanol on acetanilide disposition. Age Ageing 18: 123-126, 1989.

40. Tietz NW, Shuey DF and Wekstein DR: Laboratory values in fit aging individuals - sexagenarians through centenarians. Clin Chem 38: 1167-1185, 1992.

41. Mahmood S, Kawanaka M, Kamei A, et al: Immunohistochemical evaluation of oxidative stress markers in chronic hepatitis C. Antioxid Redox Signal 6: 19-24, 2004.

42. Stadtman ER: Importance of individuality in oxidative stress and aging. Free Radic Biol Med 33: 597-604, 2002.

43. Hirokawa K, Utsuyama M, Zeng YX, Kurashima C and Michiyuki K: Immunological alterations with aging - laying a stress on recent progress in Japan. Arch Gerontol Geriatr 19: 171-183, 1994.

44. Shimizu I, Yasuda M, Mizobuchi Y, et al: Suppressive effect of oestradiol on chemical hepatocarcinogenesis in rats. Gut 42: 112-119, 1998.

45. Shimizu I, Inoue H, Yano M, et al: Estrogen receptor levels and lipid peroxidation in hepatocellular carcinoma with hepatitis $\mathrm{C}$ virus infection. Liver 21: 342-349, 2001. 